\title{
Artículo
}

C. Moreno Lorenzo ${ }^{2}$

B. Esteban Moreno ${ }^{2}$

M.C. García Ríos ${ }^{1}$

M.J. Fernández Fernández ${ }^{2}$

C. Villaverde Gutierrez ${ }^{3}$

R. Guisado Barrilao ${ }^{3}$

${ }^{1}$ Profesor Asociado. ${ }^{2}$ Profesor Titular de Escuela Universitaria.

${ }^{3}$ Catedrático de Escuela

Universitaria. Escuela Universitaria

de Ciencias de la Salud.

Universidad de Granada.
Correspondencia:

Carmen Moreno Lorenzo

Escuela Universitaria de Ciencias

de la Salud

Avenidad de Madrid, s/n

18071 Granada

E-mail: carmenml@ugr.es

\section{Exploración y tratamiento fisioterapéutico de la hiperhidrosis palmar}

Exploration and physiotherapy treatment of palm hyperhidrosis

\section{RESUMEN}

En este artículo, se estudian las técnicas de valoración más utilizadas en la exploración de la hiperhidrosis palmar. Así mismo se describe detalladamente la técnica fisioterapéutica aplicada en la citada afección, mediante iontoforesis por agua corriente (del grifo), con distintas guías terapéuticas, lo que permite una mayor versatilidad en relación a la idiosincrasia de los pacientes.

\section{PALABRAS CLAVES}

Fisioterapia; Hiperhidrosis; Electroterapia; Iontoforesis.

\section{ABSTRACT}

This paper deals with the most used valuation techniques for palm hyperhidrosis exploration. Moreover, physiotherapy techniques applied to the treatment of this illness are detailed, this treatment being based on iontophoresis by current water with different therapeutic guides that allow more versality regarding patient idiosyncrasy.

\section{KEY WORDS}

Physiotherapy; Hyperhidrosis; Electrotherapy;

Iontophoresis. 
C. Moreno Lorenzo

B. Esteban Moreno

M.C. García Ríos

M.J. Fernández Fernández

C. Villaverde Gutierrez

R. Guisado Barrilao

106

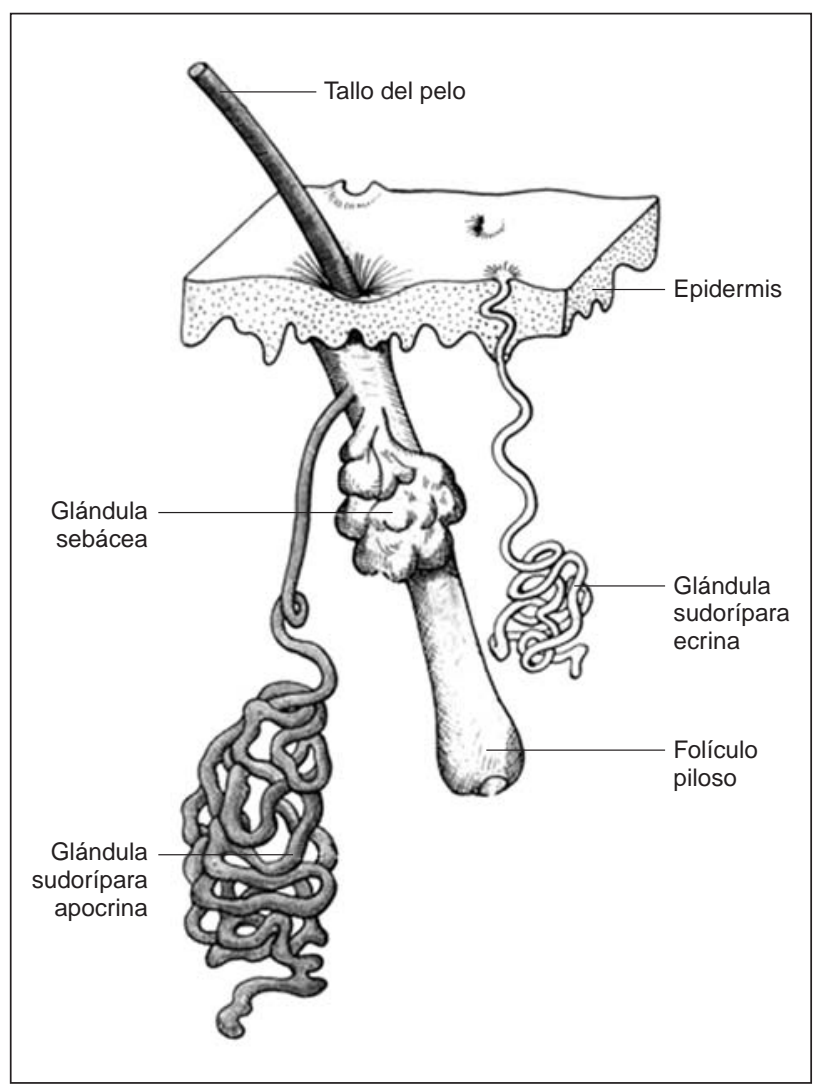

Fig. 1. Glándulas sudoríparas y folículo pilosebáceo.

\section{LA PIEL. GENERALIDADES}

La piel, uno de los órganos más grande del organismo con el $16 \%$ de su peso, se encuentra situada entre el límite corporal y el medio ambiente, desempeñando un papel importante en la termorregulación y sirviendo como fuente de información somatoestésica y por tanto como órgano efector para controlar la pérdida de calor del cuerpo ${ }^{1}$.

La piel se desarrolla a partir del ectodermo superficial, mesodermo del dermatoma y mesénquima de la placa lateral, observándose que en cada estadío de diferenciación, se torna más especializada en cuanto a estructura y función celular ${ }^{2}$. No obstante, aunque los elementos básicos de la estructura de la piel son los mismos en todas las partes del organismo, existen sin embargo
Exploración y tratamiento fisioterapéutico de la hiperhidrosis palmar

diferencias notables en su textura, estructura y función en relación al sexo, edad y región corporal considerada entre otras ${ }^{3}$.

La piel es un órgano en constante renovación, siendo su capa basal o germinativa la responsable de la producción de nuevas células. Esta capa se encuentra en el estrato más profundo del epitelio y es el único, cuyas células desarrollan mitosis. Así en seres humanos, cuando una célula deja la capa basal, su tiempo de tránsito normal hasta el estrato córneo más externo es al menos de 14 días. Sin embargo el tránsito a través del mismo y la descamación requiere otros 14 días. Una epidermis madura de la región palmo-plantar, es un epitelio escamoso estratificado, compuesto por múltiples capas de células llamadas queratinocitos, cuyo proceso de formación se denomina queratinización y que generalmente se acompaña de una pérdida del 45-86\% de su peso en seco. Dado que es un tejido en constante renovación, el proceso debe continuar durante toda la vida ${ }^{4}$.

\section{LAS GLÁNDULAS SUDORÍPARAS}

Forman parte de los anejos de la piel (fig. 1) y se clasifican en apocrinas, ecrinas y apoecrinas (GAE). Todas ellas son productoras de sudor, si bien las (GAE), constituyen un agregado reciente a la familia de las glándulas sudoríparas, siendo descubiertas en un paciente con hiperhidrosis axilar. Aunque la glándula sudorípara apoecrina es fácilmente diferenciable de la ecrina y apocrina clásicas, la diversidad de hallazgos anatómicos estructurales y ultraestructurales de la misma sigue siendo objeto de estudio. No es por tanto, hoy día, una entidad diferenciada ${ }^{5}$.

\section{Glándulas sudoríparas ecrinas}

Las glándulas sudoríparas ecrinas son glándulas exocrinas que se abren con orificios independientes en la superficie cutánea, estando compuestas por un ovillo secretor y un conducto excretor. Durante el desarrollo embrionario, estas glándulas aparecen a los 3 meses y medio en la superficie ventral de manos y pies, a los 5 meses en la piel axilar y algunas semanas más tarde, los esbozos aparecen en otras áreas del cuerpo ${ }^{3}$. En general, 
C. Moreno Lorenzo

M.C. García Ríos

M.J. Fernández Fernández

R. Guisado Barrilao
B. Esteban Moreno

C. Villaverde Gutierrez

Exploración y tratamiento fisioterapéutico de la hiperhidrosis palmar

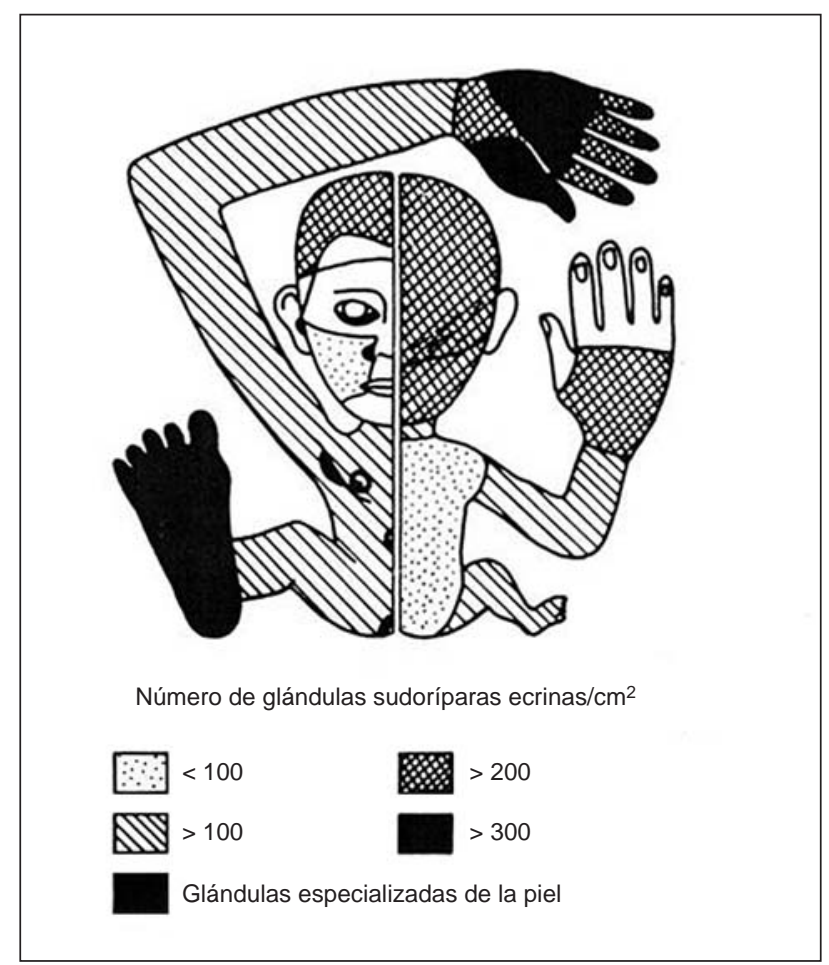

Fig 2. Distribución corporal de las glándulas sudoriparas ecrinas.

felinos en general, se encuentran en áreas tan restringidas como la pulpa digital (se duda de su eficacia como termolíticas), pero es probable que puedan facilitar en estos animales, la capacidad de adhesión de las patas durante la carrera. En el resto de los animales endotermos, tanto mamíferos como aves, no se aprecia la presencia de estas glándulas, siendo el jadeo el mecanismo termolítico más extendido. En consecuencia aunque todas las glándulas ecrinas son morfológicamente iguales, desde el punto de vista funcional, se consideran que existen dos grupos diferentes:

1. Las de las palmas de las manos y plantas de los pies, que funcionan de forma contínua y aumentan en forma notable su excreción en respuestas a estímulos mentales (intelectuales) y emocionales. Estas glándulas, como se ya se ha comentado, se desarrollan en épocas más tempranas del embrión y pueden ser análogos a glándulas similares de la superficie de apresamiento de animales inferiores.

2. El resto de las glándulas sudoríparas ecrinas, dispuestas sobre la superficie del cuerpo funcionan reac- cionando principalmente a estímulos térmicos. Además hay glándulas morfológicamente normales, que no funcionan. Las axilas contienen grupos de glándulas sudoríparas ecrinas tanto sensibles a estímulos emocionales como térmicos ${ }^{1}$. En síntesis se considera pues que existen dos modalidades de secreción sudoral ecrina, en cuanto a tipo de estimulación. Uno es la sudoración inducida por el estrés emocional (sudoración emocional), que puede tener lugar en toda la superficie cutánea en algunos individuos, pero por lo general se limita a las palmas de las manos, plantas de los pies, axilas y en algunos casos la región frontal. Este tipo de sudoración emocional se interrumpe durante el sueño. Sin embargo la sudación inducida por estímulos térmicos, continúa incluso durante el sueño si aumenta la temperatura corporal $^{1}$.

Existen diferencias notables en el grado de respuesta sudorípara a un estímulo térmico o físico dado. En ge- 
C. Moreno Lorenzo

B. Esteban Moreno

M.C. García Ríos

M.J. Fernández Fernández

C. Villaverde Gutierrez

R. Guisado Barrilao neral los hombres perspiran mas profusamente que las mujeres. El índice de sudoración en un área determinada de la piel depende de la cantidad de glándulas sudoríparas activas y del índice durante la aclimatación con distintas respuestas a nivel individual. En general aunque es el calor el principal estímulo sudoral tambien la actividad mental puede producir en determinadas circunstancias no solo sudación palmo-plantar, sino tambien en toda la superficie corporal. La actividad mental puede ser intelectual o emocional, existiendo una diversificación de respuestas de las distintas glándulas ecrinas a las distintas modalidades de estímulos mentales ${ }^{1,5}$. Tambien los estímulos gustatorios mediante la ingestión de comidas calientes y pimiento (picante) o bien otros alimentos (manzana), puede producir un aumento de la sudación como reacción fisiológica en muchas personas. Este aspecto de la sudación no es infrecuente y se produce en el 50-80\% de los pacientes sometidos a intervenciones de la glándula parótida.

\section{LA HIPERHIDROSIS}

La hiperhidrosis es una condición patológica de sobretranspiración o sobreemanación de sudor causado por la secreción excesiva de las glándulas sudoríparas ecrinas. Tambien se encuentra definida como un desorden fisiológico que provoca una sudación superior a lo normal o necesario para mantener la regulación térmica. Dado que el volumen de sudor producido ante cualquier estímulo determinado varía notablemente de persona a persona, es difícil determinar cuando el sudor es excesivo. En consecuencia, existe hiperhidrosis cuando la sudoración es clínicamente perceptible en condiciones en las que normalmente no sería apreciable o es excesiva en respuesta a los estímulos térmicos o emocionales entre otras.

En relación a la nosología de esta entidad clínica, en 1991 Sato et $\mathrm{al}^{6}$, establecen una clasificación en un intento por delimitar los distintos trastornos que afectaban a las glándulas sudoríparas ecrinas. La citada clasificación contemplaba formas secundarias y primarias así como formas generalizadas y circunscritas, encontrándose la hiperhidrosis palmar incluida en estas últimas.
Exploración y tratamiento fisioterapéutico de la hiperhidrosis palmar

\section{Hiperhidrosis palmar}

Posiblemente fue Adar et al en $1977^{7}$, quienes realizan uno de los pocos estudios sobre la hiperhidrosis, de orientación puramente epidemiológica y en este caso sobre la población de Israel, mostrando una incidencia comprendida entre el 0,6-1\%. Autores como Sato et al en $1993^{8}$, refieren que se desconoce la existencia de estadística que indiquen la frecuencia de la hiperhidrosis palmar, pero sí se puede constatar que es una afección relativamente frecuente cuando se busca y que causa una gran cantidad de problemas funcionales y emocionales a los individuos que la padecen hasta el punto de alterar el desarrollo normal de su actividad profesional, escolar y social.

Ro et al en $2002^{9}$, refieren que aunque la etiología de la hiperhidrosis es desconocida, esta tiene una incidencia aproximada del $1 \%$ en la población occidental, aunque los datos epidemiológicos son escasos e inadecuados y que son varios los investigadores que han confirmado una historia familiar positiva en pacientes tratados de hiperhidrosis. Las personas con esta afección aparentan ser perfectamente normales, sin embargo la excesiva sudación produce hipotermia por evaporación, apareciendo la piel de las manos mojadas y frías ${ }^{10}$ acompañándose en muchos casos, de lesiones eritematosas, lividez, maceración y edema ${ }^{11}$, provocando problemas funcionales y emotivos importantes. De todas las formas de presentación, es la hiperhidrosis palmar la más problemática desde el punto de vista social ya que aún cuando no es mas que una variante cuantitativa de la normalidad, crea repulsa social, disminuye la propioceptividad táctil y puede lastrar de forma grave la calidad de vida del individuo interfiriendo en sus ámbitos familiar, social y laboral así como degradar su autoestima.

\section{EXPLORACIÓN DE LA HIPERHIDROSIS EN FISIOTERAPIA}

\section{Técnicas colorimétricas. Test de Minor}

La técnica se basa en la coloración que adquiere la piel al ponerse en contacto ciertas substancias químicas con el sudor que existe en la zona de estudio. Minor en 1927 y posteriormente Wada y Takagasaki en $1948^{1}$, 
C. Moreno Lorenzo

B. Esteban Moreno

M.C. García Ríos

M.J. Fernández Fernández

C. Villaverde Gutierrez

R. Guisado Barrilao

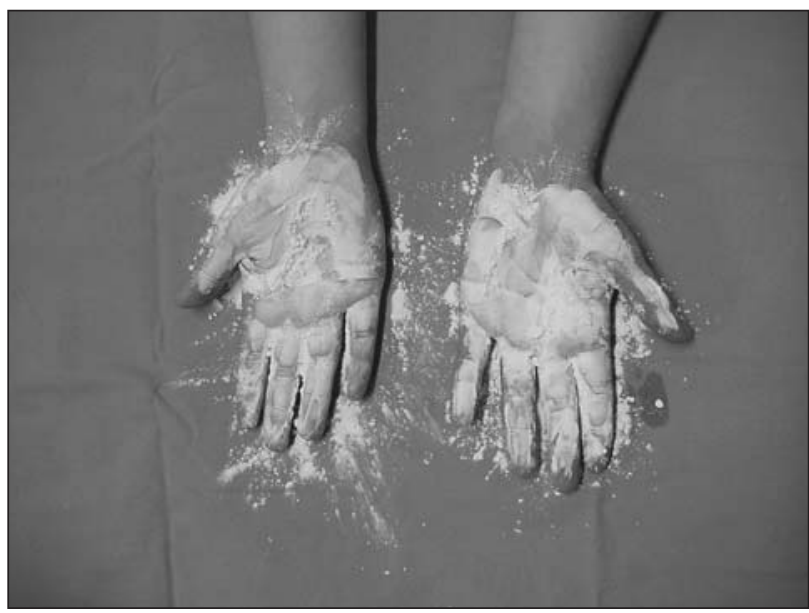

Fig. 3. Test de Minor en paciente con hiperhidrosis palmar.

pincelaron la piel de un paciente con una solución al $3 \%$ en etanol; a continuación aplicaron una mezcla de polvo de almidón en aceite de castor. El pincelado de la cara o áreas extensas del cuerpo con una solución de yodo no fue muy aceptado por los pacientes, dada la dificultad que suponía quitar la pigmentación del yodo. Por otra parte, siempre existía la posibilidad de aparición de una reacción irritante o de una absorción sistémica.

En estudios realizados en la Universidad de Granada $^{12}$, la prueba iodo-almidón de Minor, se realizó al objeto de confirmar la morfología de la hipersudación ya que el método es adecuado pues actualmente no produce reacciones irritativas y la pigmentación se retira con facilidad, amén de ofrecer excelentes contrastes que podían ser fotografiados en color.

La cara palmar de la mano se pinceló con una solución compuesta de:

$\begin{array}{ll}\text { Alcohol de } 96^{\circ}: & 500 \mathrm{ml} \\ \text { Iodo puro: } & 7,5 \mathrm{~g} \\ \text { Aceite de ricino: } & 50 \mathrm{ml}\end{array}$

Seguidamente tras una breve pausa y una vez que las zonas pinceladas se secaron, se extendió sobre ellas una capa de polvo de almidón de trigo de forma regular por la cara palmar completa (fig. 3) y se esperó unos segundos, tras los cuales se retiró la capa residual del mismo
Exploración y tratamiento fisioterapéutico de la hiperhidrosis palmar

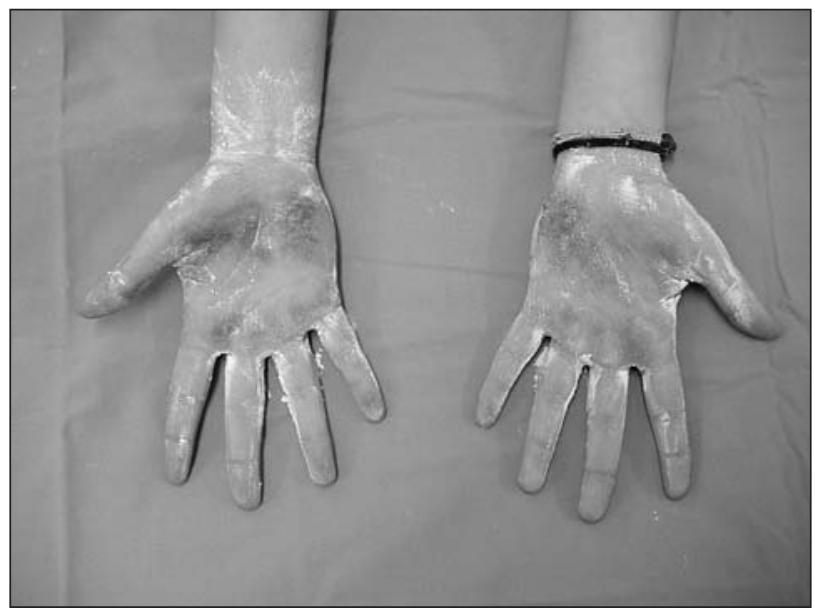

Fig. 4. Test de Minor (positivo) en paciente con hiperhidrosis palmar.

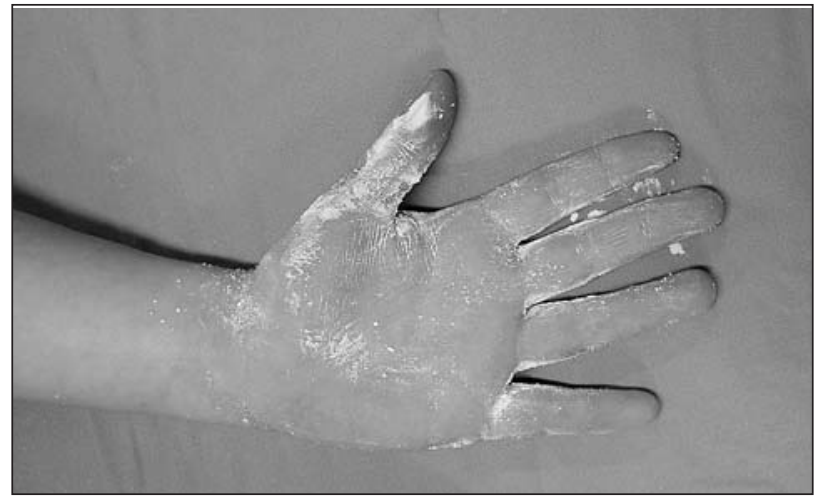

Fig. 5. Test de Minor (negativo) en paciente con euhidrosis.

observándose la aparición de la secreción sudoral, con la presencia de gotitas puntiformes oscuras en el caso de ser positivo (fig. 4), considerándose negativo el test ante la ausencia de las mismas (fig. 5).

\section{Técnicas evaporimétricas}

Se utilizan para evaluar la tasa o volumen de sudación, basándose en los cambios de presión parcial de vapor de agua en una cámara cerrada ${ }^{13}$. Kanno et $\mathrm{al}^{14}$, evaluaron objetivamente el volumen transpirado por el organismo mediante la medida del volumen de transpiración de 
C. Moreno Lorenzo

B. Esteban Moreno

M.C. García Ríos

M.J. Fernández Fernández

C. Villaverde Gutierrez

R. Guisado Barrilao

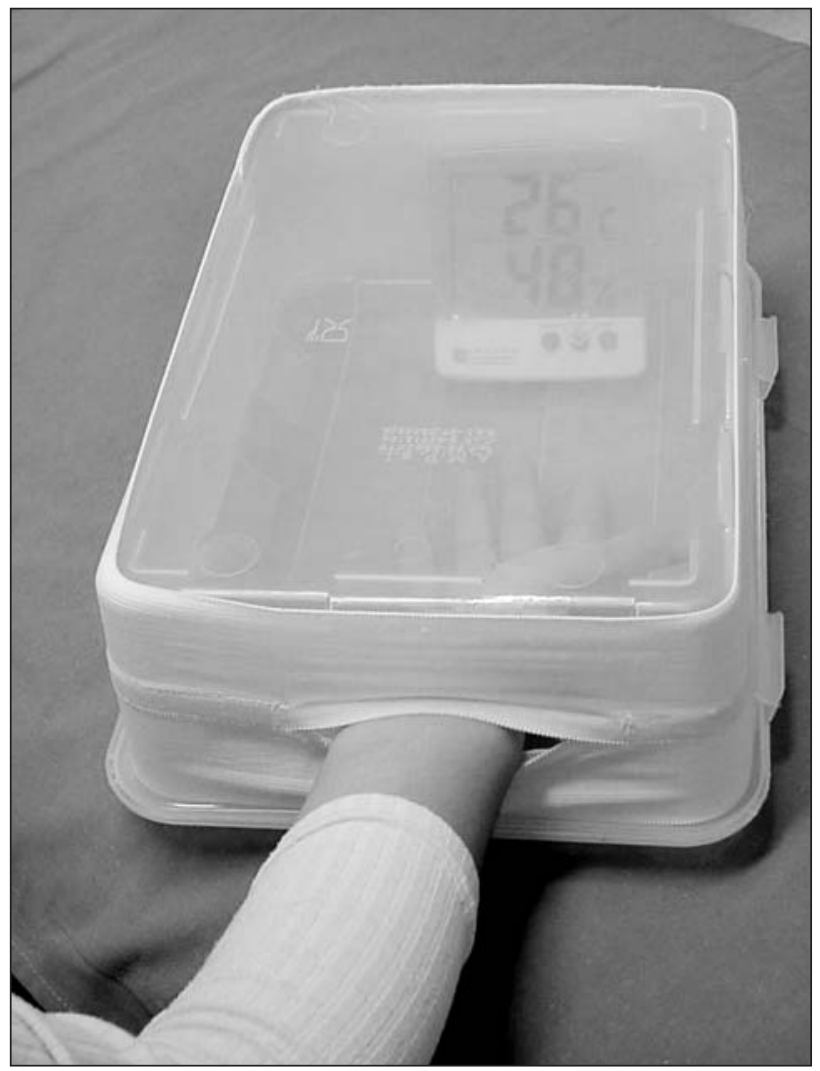

Fig. 6. Técnica evaporimétrica en paciente con hiperhidrosis.

6 pacientes con hiperhidrosis y 4 casos de hipohidrosis. Los resultados revelaron que la medida del volumen de transpiración local, con el equipo utilizado, aportó datos objetivos útiles para el diagnóstico de hiperhidrosis e hipohidrosis así como para valorar su grado.

Es evidente que las condiciones exteriores pueden influenciar la sudación líquida por una parte y por otra, la cantidad de sudor evaporada. Por lo que considerando los fundamentos sobre los que se estimaba el gradiente de presión de vapor de agua existente entre la superficie a explorar y la zona directamente adyacente a la misma, se consideró que los receptores del aparato debieran adaptarse previamente a la humedad relativa ambiental y se evitara todo movimiento de aire en la proximidad de la superficie a explorar, así como la existencia de una fuente de calor demasiado importante.
Exploración y tratamiento fisioterapéutico de la hiperhidrosis palmar

Nosotros hemos utilizado una cámara de polivinilo de doble cierre a presión (fig. 6), reforzada por malla elástica de material no transpirable y abertura elíptica virtual central para permitir la introducción de la mano del paciente en la misma. En el interior de dicha cámara se dispuso un termohigrómetro digital al objeto de medir los cambios de temperatura y humedad relativa que se producían en el interior de la misma.

\section{Técnicas gravimétricas}

Son medidas cuantitativas, determinadas mediante doble pesada en báscula de precisión del papel o gasa en las que previamente se ha empapado el sudor ${ }^{13}$.

\section{Técnicas de molde de impresión}

Existen una serie de colectores de sudor a base de materiales plásticos o silicona que se han mostrado como la mejor técnica por su fácil realización, mayor resolución y duración de los registros que pueden ser almacenados durante años, sin apenas sufrir deterioro. Esta técnica consiste en extender el material en estado fluido por la superficie de la piel, de modo que la secreción de sudor por parte de las glándulas sudoríparas deforma el material, produciendo unas impresiones permanentes cuando este se endurece. Las impresiones que coinciden con el número de glándulas sudoríparas activas, pueden ser contadas mediante amplificaciones bien sea proyectadas una vez fotografiadas o con ayuda de una lupa binocular ${ }^{15}$.

\section{Métodos electrofisiológicos}

Miden los cambios de resistencia eléctrica de la superficie cutánea debidos a la actividad secretora de las glándulas sudoríparas. Normalmente la estimulación es de tipo eléctrico y la respuesta se registra mediante electrodos cutáneos ${ }^{13}$.

Además de la hipersudación, otros aspectos clínicos que acompañan generalmente a la hiperhidrosis son los cambios cromáticos de la piel, la hipotermia y el edema, aspectos que pueden valorarse mediante una inspección codificada, termometría y perimetría respectivamente ${ }^{12}$. 
C. Moreno Lorenzo

B. Esteban Moreno

M.C. García Ríos

M.J. Fernández Fernández

C. Villaverde Gutierrez

R. Guisado Barrilao

\section{TRATAMIENTO FISIOTERAPÉUTICO DE LA HIPERHIDROSIS}

\section{Iontoforesis}

El tratamiento fisioterapéutico clásico de la hiperhidrosis palmo-plantar, consiste en iontoforesis, o técnica de facilitación del transporte de drogas moleculares a través de la piel mediante la influencia de un campo eléctrico contínuo o pulsado ${ }^{16}$.

Aunque los principios de la iontoforesis actual fueron descritos por autores como Ichiaschi en $1936^{17}$, fueron los trabajos experimentales de Leduc en 1908, los primeros en tener un carácter científico. Posteriormente, la importancia del método como alternativa de administración terapéutica, acaparó la atención de muchos investigadores entre los que podemos citar a Sing et al en $1994^{18}$ y Nair et al en $1999^{19}$, quienes profundizaron en el conocimiento de la piel como barrera limitante. Otros autores como Howard et al en $1995^{20}$ y Guffey et al en $2000^{21}$, destacaron la importancia del $\mathrm{pH}$ como variable crítica de la iontoforesis por sus repercusiones sobre la piel y el flujo electroosmótico, aspecto este último tambien estudiado por Gangarosa en $1980^{22}$. No obstante, el problema del transporte por iontoforesis y la búsqueda de modelos experimentales fué planteado por Bronaugh et $\mathrm{al}^{23}$, Monteiro-Rivière ${ }^{24}$, Pikal et $\mathrm{al}^{25}$, Banga et $\mathrm{al}^{26}$ y Kalia et $\mathrm{al}^{27}$.

Aspectos muy vinculados a la técnica electroterapéutica como la densidad, la morfología y el tiempo de aplicación de la corriente fueron especialmente estudiados por Abramson y Gorin en $1941^{16}$, quienes establecen los límites máximos de aplicación de la misma. Sin embargo, fueron Inada et al en $1994^{28}$ los que observan los cambios que el campo eléctrico produce sobre la epidermis humana y la influencia que tienen algunos factores como el voltaje y el tiempo de aplicación sobre los mismos.

El mecanismo de acción de la iontoforesis en el caso de la hiperhidrosis, ha sido ampliamente discutido por Hill et $\mathrm{al}^{29}$, Levitt ${ }^{30}$, Shelley et $\mathrm{al}^{31}$, Quinton ${ }^{32}$ y Sato et $\mathrm{al}^{8}$, observándose en todos ellos una heterogeneidad de los hallazgos. Aún considerando que el mecanismo de acción de la iontoforesis, en el caso de la hiperhidrosis palmar, sigue siendo todavía un enigma sin resolver, estudios recientes ${ }^{12}$ han objetivado que la hiperhidrosis palmar implica cambios morfológicos respecto de la normalidad y que el tratamiento por iontoforesis mas agua corriente (del grifo) permite abrigar la esperanza de una reversibilidad de los mismos a medio plazo.

Por último, la eficacia de la iontoforesis en la hiperhidrosis ha sido ampliamente demostrada por Shrivastava et $\mathrm{al}^{33}$, Levit ${ }^{34}$, Vayssairat et $\mathrm{al}^{35}$, Carpentier et $\mathrm{al}^{36}$, Raulin et $\mathrm{al}^{37}$, Haulot et $\mathrm{al}^{38}$, Wollina et $\mathrm{al}^{39}$, Rioja et $\mathrm{al}^{40}$ y Karakoc et a ${ }^{41}$ entre otros. En general, la eficacia terapéutica ha sido demostrada de forma exhaustiva con la corriente galvánica contínua, si bien autores como Reinauer et $\mathrm{al}^{42}$, muestran la ausencia de menoscabo terapéutico con corriente alterna mas corriente directa, no observándose virtualmente ningún efecto con la corriente alterna pura. Posteriormente Reinauer et al en $1995^{43}$, observaron tambien una disminución importante de los efectos electrobiológicos secundarios a esta terapéutica, con la administración de corrientes directas pulsadas de media frecuencia.

En nuestro estudio ${ }^{12}$, (fig. 7) siguiendo la línea marcada por los autores citados, confirmamos la importancia de la eficacia de la corriente galvánica contínua en la resolución de la hiperhidrosis palmar. Sin embargo, los objetivos a conseguir en el paciente hiperhidrótico nos ha conducido a combinar o alternar la corriente clásica con corriente directa pulsada de $10 \mathrm{KHz}$, de dosis globales con rango de 10-30 mA, a tiempos totales de 20-30 minutos con alter-

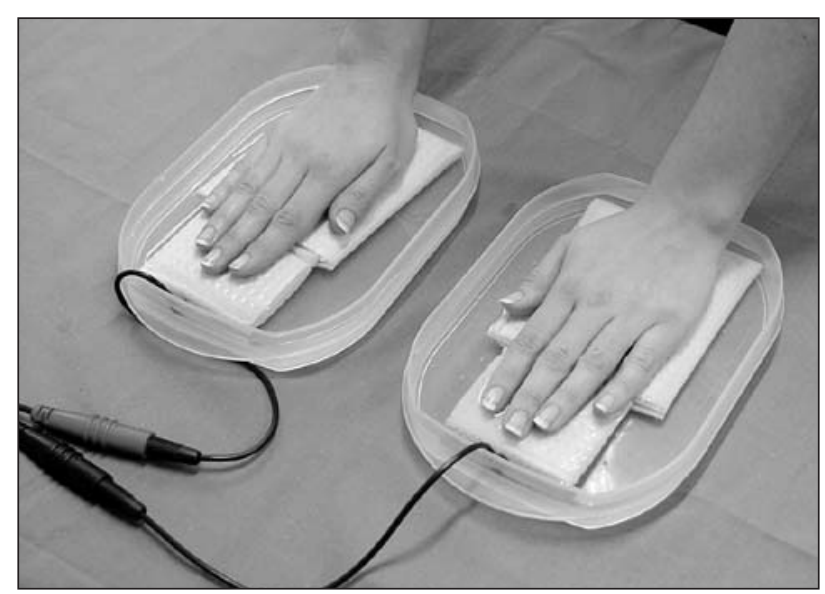

Fig. 7. Tratamiento de la hiperhidrosis palmar por iontoforesis más agua corriente. 
C. Moreno Lorenzo

B. Esteban Moreno

M.C. García Ríos

M.J. Fernández Fernández

C. Villaverde Gutierrez

R. Guisado Barrilao
Exploración y tratamiento fisioterapéutico de la hiperhidrosis palmar
112 nancia de la polaridad y a sectorización de los tiempos en relación a las dosis de aplicación, (apoyándonos en los fundamentos de Inada et $\mathrm{al}^{28}$ ). Así mismo, aunque los rangos de densidad de corriente han estado comprendidos entre $0,18-0,31 \mathrm{~mA} / \mathrm{cm}^{2}$, se ha pretendido aumentar la misma en las zonas afectas, mediante la aplicación de productos petrolados, siguiendo a Sato et $\mathrm{al}^{8}$.

Dado que los objetivos a conseguir para el paciente han sido principalmente la obtención de: una mínima fase terapéutica, un máximo período de remisión sudoral, mínimos efectos electrobiológicos y máxima calidad de vida, las circunstancias nos ha exigido priorizar en cada paciente y en cada caso, los objetivos reseñados, considerando no solo la tolerancia del paciente a la actividad terapéutica, sino tambien la obtención de unos resultados lo más compatibles con su calidad de vida, apoyándonos en el cuestionario Dermatology Life Quality Index (DLQI) ${ }^{44}$.

\section{BIBLIOGRAFÍA}

1. Camacho-Martínez F. Enfermedades de los anejos cutáneos. En: De Dulanto F, Armijo M, Camacho-Martínez F, Naranjo R. Dermatología Médio-Quirúrgica. Vol. II. Granada: Anel, 1982; p. 1001-100.

2. Thibodeau GA, Patton KT. Anatomía y fisiología. Estructura y función del cuerpo humano. 2. ed. Madrid: Mosby/Doyma Libros, 1995.

3. Sadler TW. Langman. Embriología médica. Con orientación clínica. 8ª ed. Madrid: Panamericana, 2001.

4. Fawcet DW. Tratado de histología. 12.a ed. Madrid: Mc Graw-Hill/Interamericana de España, 1997.

5. Freedberg IM, Eisen AZ, Wolf K, Austen KF, Golddsmith LA. Katz SI, Fitzpatrick TB. Fitzpatrick. Dermatología en Medicina General. Tomo I, 5a ed. Madrid: Panamericana, 2001.

6. Sato K, Otsuyama M, Samman G. Eccrine sweat glands disorders. J Am Acad Dermatol 1991;24(6 Pt 1):1010-4.

7. Adar R, Kurchin A, Zweig A, Mozes M. Palmar hyperhidrosis and its surgical treatment: a report of 100 cases. Ann Surg 1977;186(1):34-41.

8. Sato K, Timm DE, Sato F, Templeton EA, Meletiou DS, Toyomoto T, Soos G, Sato SK. Generation and transit pathway of $\mathrm{H}^{+}$is critical for inhibition of palmar sweating by intophoresis in water. J Appl Physiol 1993;75(5):2258-64.

9. Ro KM, Cantor RM, Lange KL, Ahn SS. Palmar hyperhidrosis:Evidence of genetic transmission. J Vasc Sug 2002;35(2): 382-6.

10. Sato K, Kang WH, Saga K, Sato KT. Biology of sweat glands and their disorders.II.Disorders of sweat gland function.J Am Acad Dermatol 1989;20(5Pt 1):713-26.

11. Holzle E, Alberti N. Long-term efficacy and side effects off tap water iontophoresis of palmoplantar hyperhidrosis - the usefulness of home therapy. Dermatologica 1987;175(3):126-35.
12. Moreno C. Estudio clínico-fisioterapéutico comparativo mediante tres modalidades de electroterapia en el tratamiento de la hiperhidrosis. Tesis Doctoral. Granada: Universidad de Granada. Escuela Universitaria de Ciencias de la Salud, 2003.

13. Naver H, Swartling C, Aquilonius SM. Palmar and axillary treated with botulinum toxin:one year clinical follow-up. Eur J Neurol 2000;7(1):55-62.

14. Kanno K, Yohiike T. Quantitative evaluation of perspiration with equipment for continuous recording of local perspiration volumen (II): Hyperhidrosis and hipohidrosis. Nippon Hifuke Gakkai Zasshiu 1989;99(11):1153-7.

15. Callejas MA, Serra M. Hiperhidrosis primitiva: diagnóstico y tratamiento. Jano, Med humanid 2000;59(1352):41-3.

16. Rioja J. Usos terapéuticos de la corriente galvánica: galvanismo médico e iontoforesis. Valladolid: Insalud, 1995.

17. Ichiaschi T. Effect of drugs on the sweat glands by cataphoresis and an effective retics and adiaphoretic. J Oriental Med 1936;25:101-2.

18. Sing P, Maibach HI. Iontophoresis in drug delivery: basic principles and applications. Crit Rev Ther Drug Carrier Syst 1994;11(2-3):161-213.

19. Nair V, Pillai O, Poduri R. Panchagnula R. Transdermal iontophoresis. Part I:Basic principles and considerations. Methods Find Exp Clin Pharmacol 199;21(2):139-51.

20. Howard JP, Drake TR, Kellogg DL Jr. Effects of alternating current iontophoresis on drug delivery. Arch Phys Med Rehabil 1995;76(5):463-6.

21. Guffey JS, Rutherford MJ, Payne W, Phillips C. Skin pH changes associated with iontophoresis. J Orthop Sports Phys Ther 2000;(3082):109.

22. Gangarosa LP, Park NH, Wiggins CA, Hill JM. Increased penetration of non electroytes into mouse skin during iontophore- 
C. Moreno Lorenzo

B. Esteban Moreno

M.C. García Ríos

M.J. Fernández Fernández

C. Villaverde Gutierrez

R. Guisado Barrilao
Exploración y tratamiento fisioterapéutico de la hiperhidrosis palmar

35. Vayssairat M, Cormier JM, Priollet P, Bourdin JP, Brun JP, Housset E. L ionization doit-elle remplacer la sympathectomie dans le traitement de 1 hyperhidrose palmo-plantaire? Pr Med 1983;12(5):301-2.

36. Carpentier P, Drevet JG, Dousson D, Bouchet JY, Richaud C, Franco A. Interêt du courant galvanique dans le traitement de 1 hyperhidrose palmaire. En: Simon L. Actualités en Rééducation Fonctionnelles et Réadaptation. 10 ${ }^{\mathrm{e}}$ série. Paris: Masson, 1985; p.44-9.

37. Raulin C, Rosing S, Petzoldt D. Home treatment of hyperhidrosis of the hands and feet tap water iontophoresis. Hautarzt 1988;39(8):504-8

38. Haulot A, Chiesa G, Menager D. Traitement de 1 hyperhidrose du moignon par iontophorèse: a propos de 31 cas. En: Simon L, Pélisier J, Hérrison C. Actualités en Réeducation Fonctionnelle et Réadaptation. $17^{\mathrm{e}}$ série. Paris: Masson, 1992; p.100-4.

39. Wollina U, Uhlemann C, Elstermann D, Kober L, Barta U. Therapy of hyperhidrosis with iontophoresis. Positive effect on healing time and lack of recurrence in hand-foot eczema. Hautarzt 1998;49(2):109-13.

40. Rioja J, Cantalapiedra E, Room M, González A, Prada J. Tratamiento iontoforético de la hiperhidrosis palmoplantar. Rehabilitación (Madr) 2001;35(4):219-24.

41. Karakoc Y, Aydemir EH, Kalkan MY, Unal G. Safe control of palmoplantar hyperhidrosis with direct electrical current. Int J Dermatol 2002;41(9):602-5.

42. Reinauer S, Neusser A, Schauf G, Holzle E. Iontophoresis with alternating current and direct current offset (AC/DC iontophoresis): a new approach for the treatment of hyperhidrosis. $\mathrm{Br} \mathrm{J}$ Dermatol 1993;129(2):166-9.

43. Reinauer S, E. Pulsed direct current iontophoresis as a possible new treatment for hyperhidrosis. Hautartz 1995;46(8):543-7.

44. De Tiedra AG, Mercadal J, Badia X, Mascaro JM, Herdman M, Lozano R. Adaptación transcultural al español del cuestionario Dermatology Life Quality Index (DLQI): el Índice de Calidad de Vida en Dermatología. Actas Dermosifiliog 1998;89(12): 692-700.

34. Levit F. Treatment of hyperhidrosis by tap water iontophoresis.Cutis 1980;26(2):192-4.

\section{FE DE ERRATAS}

En el fascículo I (volumen 26), de la revista Fisioterapia en las páginas correspondientes al sumario, tanto en castellano como en inglés, hay el siguiente error: en el artículo "Precisión del eje sagital según el método de medición por plomada", se incluyen cinco autores, de los cuales, los dos primeros (R. Fernández Cervantes y S. Patiño Núñez), no son autores del mismo (como puede observarse en la página I3). 